\title{
Tungsten Alkyl Alkylidyne and Bis-alkylidene Complexes. \\ Preparation and Kinetic and Thermodynamic Studies of Their Unusual Exchanges
}

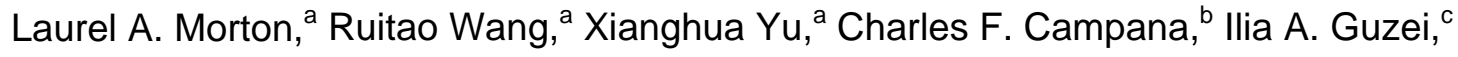
Glenn P. A. Yap, ${ }^{d}$ Zi-Ling Xue ${ }^{\star, a}$

a Department of Chemistry, The University of Tennessee, Knoxville, Tennessee 37996

${ }^{b}$ Advanced X-ray Solutions, Bruker AXS Inc., Madison, Wisconsin 53711

${ }^{\mathrm{c}}$ Department of Chemistry, The University of Wisconsin, Madison, Wisconsin 53706

${ }^{\mathrm{d}}$ Department of Chemistry \& Biochemistry, The University of Delaware, Newark, DE 19716

\section{Supporting Information}



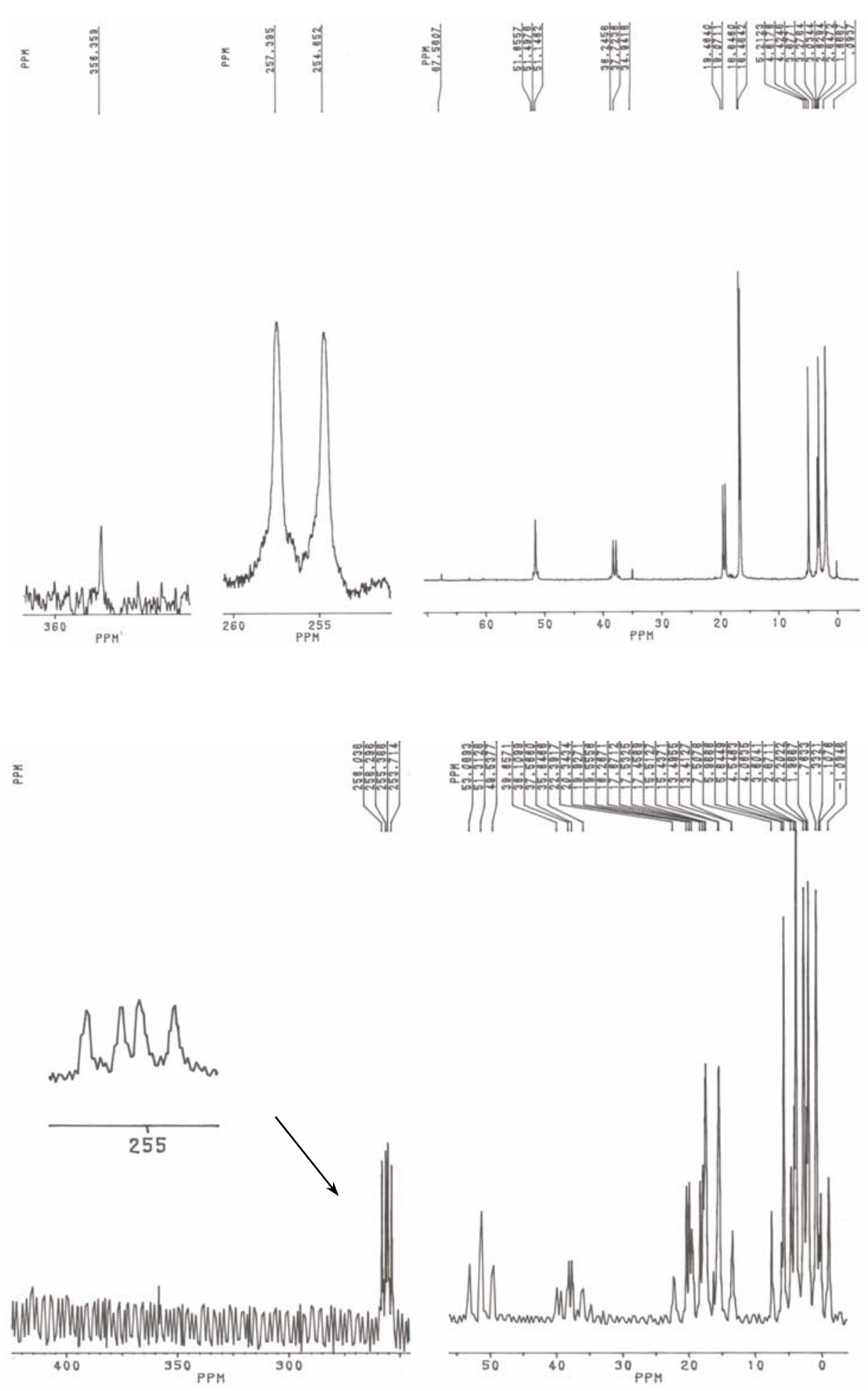

Figure S1. Upper: ${ }^{13} \mathrm{C}$ NMR $(62.896 \mathrm{MHz})$ spectrum of a mixture of $\mathbf{1 a}$ and $\mathbf{1 b}$ at $23^{\circ} \mathbf{C}$. Lower: ${ }^{1} \mathrm{H}$-gated-decoupled ${ }^{13} \mathrm{C}$ NMR $(62.896 \mathrm{MHz})$ spectrum of a mixture of $\mathbf{1 a}$ and $\mathbf{1 b}$ at $23^{\circ} \mathrm{C}$. 

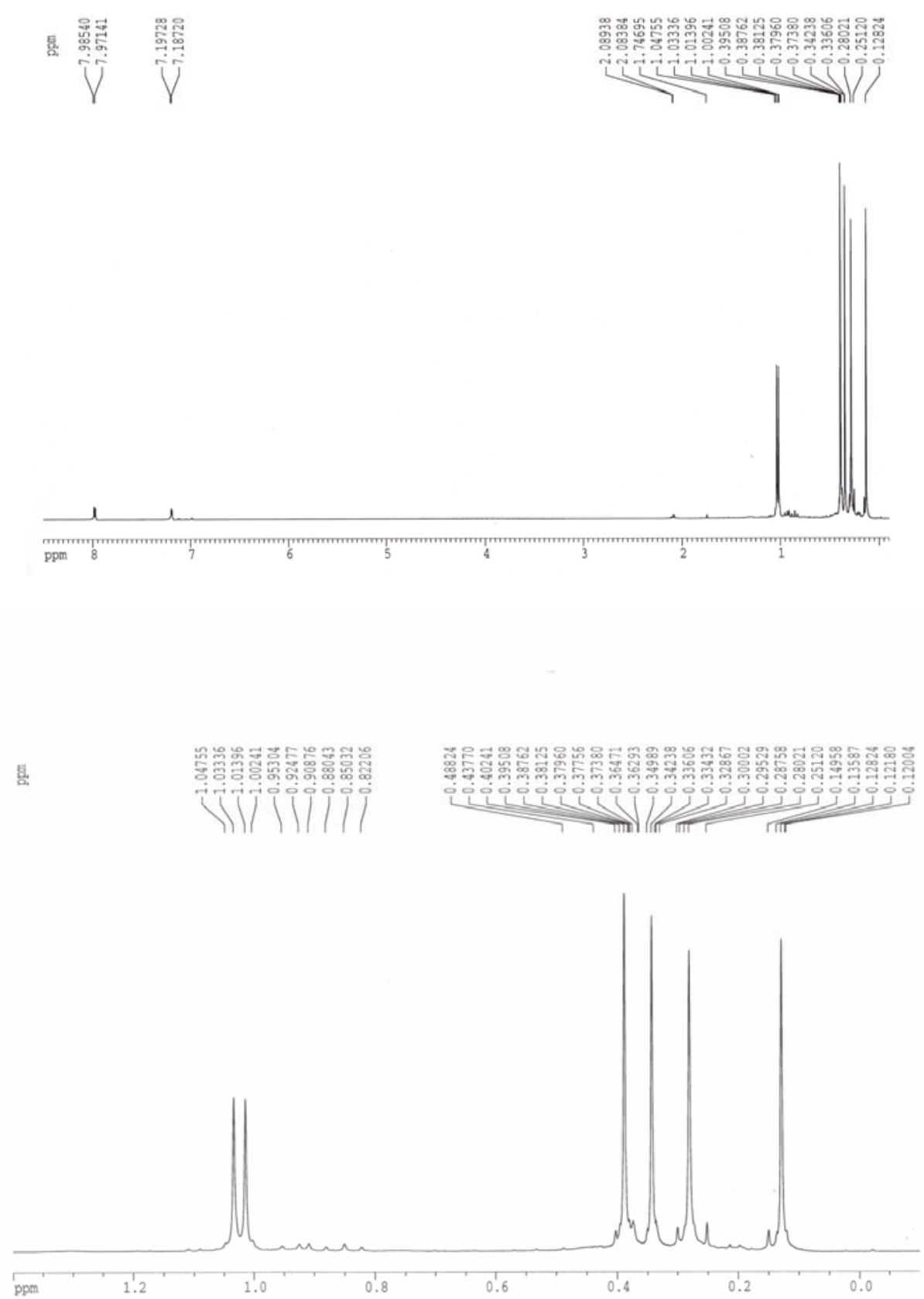

Figure S2. Upper: ${ }^{1} \mathrm{H}$ NMR (400.11 MHz) spectrum of $\left(\mathrm{Me}_{3} \mathrm{SiCH}_{2}\right)_{2} \mathrm{~W}(=\mathrm{CHSiMe})_{2}\left(\mathrm{PMe}_{3}\right)$ (1) $)$ at $-20^{\circ} \mathrm{C}$. Lower: Expansion of the $-0.1-1.4 \mathrm{ppm}$ region of the spectrum. 

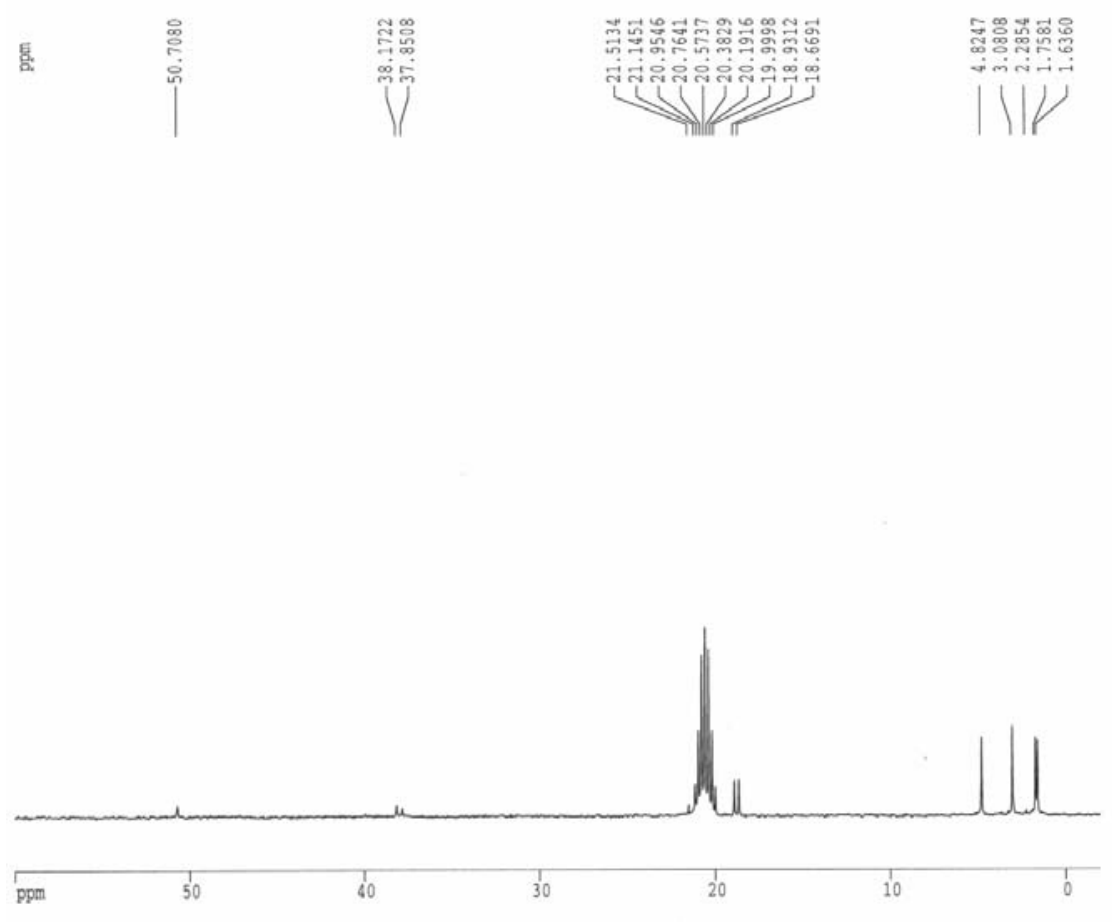

5.

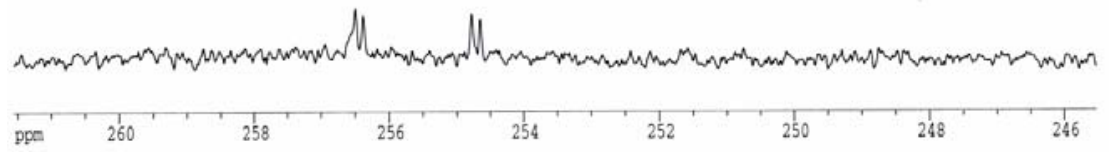

Figure S3. ${ }^{13} \mathrm{C}$ NMR $(100.63 \mathrm{MHz})$ spectrum of $\mathbf{1 b}$ at $-40{ }^{\circ} \mathrm{C}$. Upper: $-2-60 \mathrm{ppm}$ region. Lower: 246-261 ppm region. 


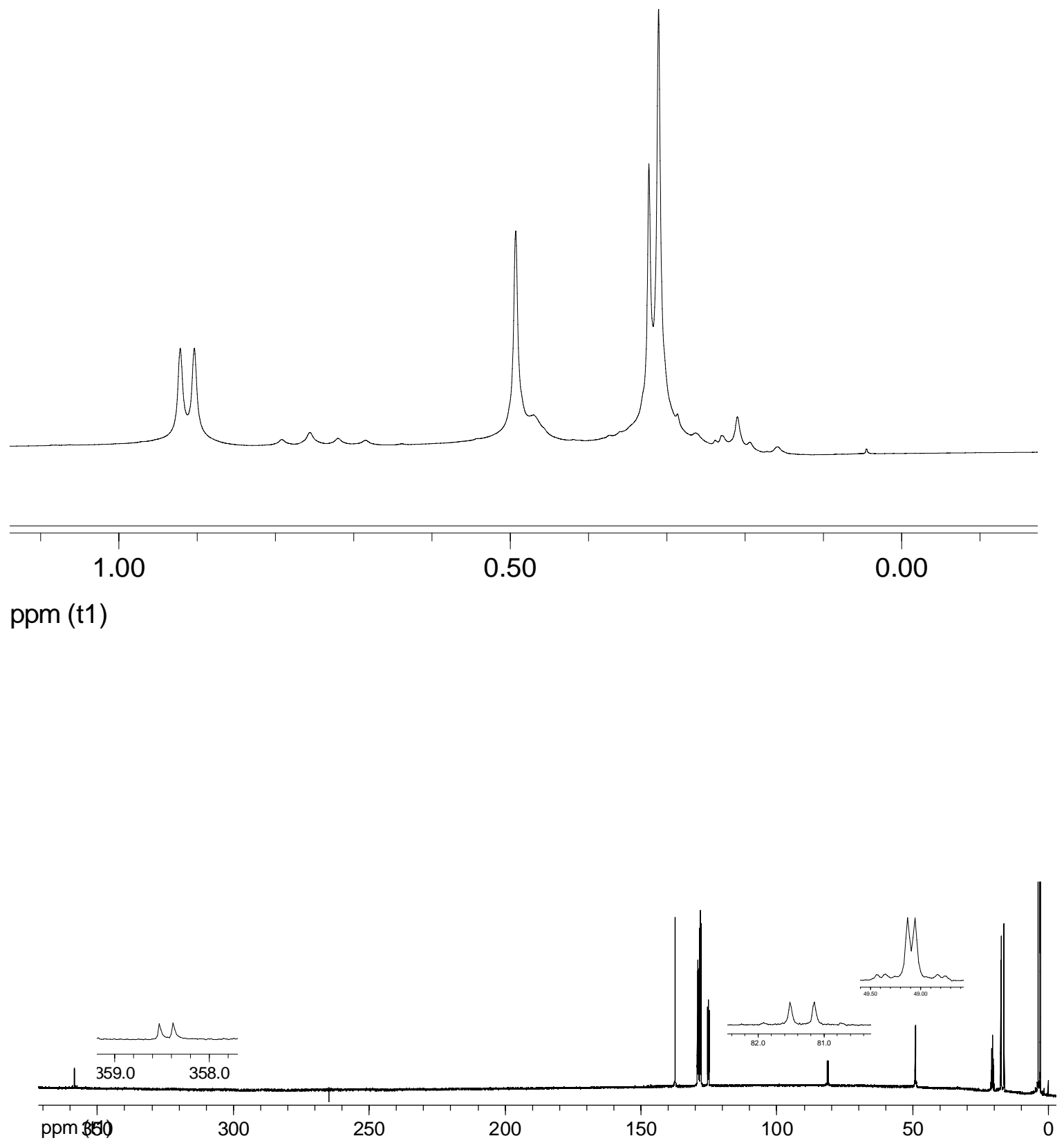

Figure S4. Upper: ${ }^{1} \mathrm{H}$ NMR $(400.11 \mathrm{MHz})$ spectrum of $1 \mathbf{a}$ at $-50{ }^{\circ} \mathrm{C}$.

Lower: ${ }^{13} \mathrm{C}$ NMR $(100.63 \mathrm{MHz})$ of $1 \mathrm{a}$ at $-50^{\circ} \mathrm{C}$. 
Table S1. Crystal data and structure refinement for $\mathbf{1 b}$.

\begin{tabular}{|c|c|c|}
\hline Empirical formula & C19 H53 P Si4 W & \\
\hline Formula weight & 608.79 & \\
\hline Temperature & $173(2) \mathrm{K}$ & \\
\hline Wavelength & $0.71073 \AA$ & \\
\hline Crystal system & Hexagonal & \\
\hline Space group & P6(3) & \\
\hline Unit cell dimensions & $a=12.1310(5) \AA$ & $\alpha=90^{\circ}$ \\
\hline & $\mathrm{b}=12.1310(5) \AA$ & $\beta=90^{\circ}$ \\
\hline & $c=12.1091(5) \AA$ & $\gamma=120^{\circ}$ \\
\hline Volume & $1543.25(11) \AA^{3}$ & \\
\hline Z & 2 & \\
\hline Density (calculated) & $1.310 \mathrm{Mg} / \mathrm{m}^{3}$ & \\
\hline Absorption coefficient & $3.953 \mathrm{~mm}^{-1}$ & \\
\hline$F(000)$ & 624 & \\
\hline Crystal size & $0.35 \times 0.25 \times 0.25$ & \\
\hline Theta range for data collection & 1.68 to $27.42^{\circ}$ & \\
\hline Index ranges & $-15 \leq h \leq 15,-15 \leq$ & $15,-15 \leq \mathrm{I} \leq 15$ \\
\hline Reflections collected & 15949 & \\
\hline Independent reflections & $2352[R$ (int) $=0.03$ & \\
\hline Completeness to theta $=27.42^{\circ}$ & $100.0 \%$ & \\
\hline Absorption correction & Semi-empirical fro & quivalents \\
\hline Max. and min. transmission & 0.6850 and 0.622 & \\
\hline Refinement method & Full-matrix least-sc & es on $\mathrm{F}^{2}$ \\
\hline Data / restraints / parameters & 2352 / 112 / 86 & \\
\hline Goodness-of-fit on $\mathrm{F}^{2}$ & 1.205 & \\
\hline Final $R$ indices $[I>2 \operatorname{sigma}(I)]$ & $\mathrm{R} 1=0.0624, \mathrm{wR} 2$ & 1233 \\
\hline $\mathrm{R}$ indices (all data) & $\mathrm{R} 1=0.0654, \mathrm{wR} 2$ & 1253 \\
\hline Absolute structure parameter & $0.44(11)$ & \\
\hline Largest diff. peak and hole & 1.878 and -2.405 & \\
\hline
\end{tabular}


Table S2. Atomic coordinates $\left(\times 10^{4}\right)$ and equivalent isotropic displacement parameters $\left(\AA^{2} \times 10^{3}\right)$ for $\mathbf{1 b} . \mathrm{U}(\mathrm{eq})$ is defined as one third of the trace of the orthogonalized $\mathrm{Uij}$ tensor.

\begin{tabular}{|c|c|c|c|c|}
\hline & $x$ & $y$ & $z$ & $\mathrm{U}(\mathrm{eq})$ \\
\hline$\overline{W(1)}$ & 3333 & 6667 & 9210(1) & 59(1) \\
\hline$P(1)$ & 3333 & 6667 & 11286(4) & 63(1) \\
\hline$C(1)$ & $3860(20)$ & $8238(18)$ & $11940(20)$ & $104(8)$ \\
\hline$C(2)$ & $5036(11)$ & $8174(11)$ & 9332(15) & $71(4)$ \\
\hline $\operatorname{Si}(1)$ & $6694(4)$ & $8485(3)$ & $9183(7)$ & $65(1)$ \\
\hline$C(3)$ & $7450(20)$ & $9390(20)$ & 7899(16) & 106(8) \\
\hline C(4) & $7650(30)$ & $9438(18)$ & 10393(17) & 108(9) \\
\hline C(5) & $6736(14)$ & $6956(11)$ & $9150(20)$ & $112(6)$ \\
\hline$C(6)$ & $2550(30)$ & $6480(40)$ & $7679(14)$ & 69(10) \\
\hline $\mathrm{Si}(2)$ & 3333 & 6667 & $6287(4)$ & $64(1)$ \\
\hline$C(7)$ & $4740(20)$ & $6460(30)$ & $6470(30)$ & 39(9) \\
\hline$C(8)$ & $3910(40)$ & $8350(20)$ & $5860(50)$ & $300(100)$ \\
\hline C(9) & $2270(30)$ & $5590(30)$ & $5200(30)$ & $95(16)$ \\
\hline
\end{tabular}


Table S3. Bond lengths $(\AA)$ and angles $\left(^{\circ}\right)$ for $\mathbf{1 b}$

\begin{tabular}{|c|c|c|c|}
\hline $\mathrm{W}(1)-\mathrm{C}(2) \# 1$ & $1.963(12)$ & $\mathrm{Si}(2)-\mathrm{C}(9)$ & $1.850(15)$ \\
\hline$W(1)-C(2)$ & $1.963(12)$ & Si(2)-C(9)\#2 & $1.850(15)$ \\
\hline$W(1)-C(2) \# 2$ & $1.963(12)$ & Si(2)-C(9)\#1 & $1.850(15)$ \\
\hline$W(1)-C(6)$ & $2.042(18)$ & $\operatorname{Si}(2)-C(6) \# 1$ & $1.890(18)$ \\
\hline$W(1)-C(6) \# 1$ & $2.043(18)$ & Si(2)-C(6)\#2 & $1.890(19)$ \\
\hline$W(1)-C(6) \# 2$ & $2.043(18)$ & Si(2)-C(8)\#1 & $1.874(16)$ \\
\hline $\mathrm{W}(1)-\mathrm{P}(1)$ & $2.514(5)$ & $\mathrm{Si}(2)-\mathrm{C}(8)$ & $1.874(15)$ \\
\hline$P(1)-C(1) \# 1$ & $1.86(2)$ & $\operatorname{Si}(2)-C(8) \# 2$ & $1.874(15)$ \\
\hline$P(1)-C(1)$ & $1.86(2)$ & $\operatorname{Si}(2)-C(7) \# 2$ & $1.855(15)$ \\
\hline$P(1)-C(1) \# 2$ & $1.86(2)$ & $\mathrm{Si}(2)-\mathrm{C}(7)$ & $1.855(15)$ \\
\hline$C(2)-\operatorname{Si}(1)$ & $1.861(13)$ & Si(2)-C(7)\#1 & $1.855(15)$ \\
\hline $\mathrm{Si}(1)-\mathrm{C}(4)$ & 1.868(13) & $C(7)-C(8) \# 2$ & $0.83(7)$ \\
\hline $\mathrm{Si}(1)-\mathrm{C}(3)$ & $1.861(13)$ & $C(7)-C(9) \# 1$ & $1.64(5)$ \\
\hline $\mathrm{Si}(1)-\mathrm{C}(5)$ & 1.881(10) & $C(7)-C(6) \# 1$ & $1.97(4)$ \\
\hline$C(6)-C(6) \# 2$ & $1.48(4)$ & $C(8)-C(7) \# 1$ & $0.83(7)$ \\
\hline$C(6)-C(6) \# 1$ & $1.48(4)$ & $C(8)-C(9) \# 2$ & $1.09(7)$ \\
\hline $\mathrm{C}(6)-\mathrm{Si}(2)$ & $1.890(18)$ & $C(9)-C(8) \# 1$ & $1.09(7)$ \\
\hline$C(6)-C(7) \# 2$ & $1.97(4)$ & $C(9)-C(7) \# 2$ & $1.64(5)$ \\
\hline$C(2) \# 1-W(1)-C(2)$ & $119.44(14)$ & $C(1)-P(1)-W(1)$ & $115.4(8)$ \\
\hline$C(2) \# 1-W(1)-C(2) \# 2$ & $119.44(15)$ & $\mathrm{C}(1) \# 2-\mathrm{P}(1)-\mathrm{W}(1)$ & $115.4(8)$ \\
\hline$C(2)-W(1)-C(2) \# 2$ & $119.44(14)$ & $\mathrm{Si}(1)-C(2)-W(1)$ & $135.1(7)$ \\
\hline$C(2) \# 1-W(1)-C(6)$ & $70.9(8)$ & $C(2)-S i(1)-C(4)$ & $108.9(12)$ \\
\hline$C(2)-W(1)-C(6)$ & $112.6(12)$ & $C(2)-S i(1)-C(3)$ & 111.3(8) \\
\hline$C(2) \# 2-W(1)-C(6)$ & $98.5(14)$ & $C(4)-S i(1)-C(3)$ & 108.6(6) \\
\hline$C(2) \# 1-W(1)-C(6) \# 1$ & 112.6(11) & $C(2)-S i(1)-C(5)$ & $111.2(6)$ \\
\hline$C(2)-W(1)-C(6) \# 1$ & $98.5(14)$ & $C(4)-S i(1)-C(5)$ & 108.1(7) \\
\hline$C(2) \# 2-W(1)-C(6) \# 1$ & $70.9(8)$ & $C(3)-S i(1)-C(5)$ & $108.7(7)$ \\
\hline$C(6)-W(1)-C(6) \# 1$ & $42.6(11)$ & $C(6) \# 2-C(6)-C(6) \# 1$ & $59.999(16)$ \\
\hline$C(2) \# 1-W(1)-C(6) \# 2$ & $98.5(14)$ & C(6)\#2-C(6)-Si(2) & $66.9(6)$ \\
\hline$C(2)-W(1)-C(6) \# 2$ & $70.9(8)$ & C(6)\#1-C(6)-Si(2) & $66.9(6)$ \\
\hline$C(2) \# 2-W(1)-C(6) \# 2$ & $112.6(11)$ & $C(6) \# 2-C(6)-C(7) \# 2$ & $123.0(13)$ \\
\hline$C(6)-W(1)-C(6) \# 2$ & $42.6(11)$ & $C(6) \# 1-C(6)-C(7) \# 2$ & $87(3)$ \\
\hline$C(6) \# 1-W(1)-C(6) \# 2$ & $42.6(11)$ & $\mathrm{Si}(2)-C(6)-C(7) \# 2$ & $57.4(9)$ \\
\hline$C(2) \# 1-W(1)-P(1)$ & $85.7(5)$ & $C(6) \# 2-C(6)-W(1)$ & $68.7(5)$ \\
\hline$C(2)-W(1)-P(1)$ & $85.7(5)$ & $\mathrm{C}(6) \# 1-\mathrm{C}(6)-\mathrm{W}(1)$ & $68.7(5)$ \\
\hline$C(2) \# 2-W(1)-P(1)$ & $85.7(5)$ & $\mathrm{Si}(2)-\mathrm{C}(6)-\mathrm{W}(1)$ & $128.2(12)$ \\
\hline$C(6)-W(1)-P(1)$ & $155.2(6)$ & $\mathrm{C}(7) \# 2-\mathrm{C}(6)-\mathrm{W}(1)$ & $143(2)$ \\
\hline$C(6) \# 1-W(1)-P(1)$ & $155.2(6)$ & C(9)-Si(2)-C(9)\#2 & $75(2)$ \\
\hline$C(6) \# 2-W(1)-P(1)$ & $155.2(6)$ & C(9)-Si(2)-C(9)\#1 & $75(2)$ \\
\hline$C(1) \# 1-P(1)-C(1)$ & 103.0(9) & $\mathrm{C}(9) \# 2-\mathrm{Si}(2)-\mathrm{C}(9) \# 1$ & $75(2)$ \\
\hline $\mathrm{C}(1) \# 1-\mathrm{P}(1)-\mathrm{C}(1) \# 2$ & 103.0(9) & $\mathrm{C}(9)-\mathrm{Si}(2)-\mathrm{C}(6) \# 1$ & $123(2)$ \\
\hline$C(1)-P(1)-C(1) \# 2$ & 103.0(9) & $\mathrm{C}(9) \# 2-\mathrm{Si}(2)-\mathrm{C}(6) \# 1$ & 161.1(16) \\
\hline$C(1) \# 1-P(1)-W(1)$ & $115.4(8)$ & C(9)\#1-Si(2)-C(6)\#1 & $115.0(15)$ \\
\hline
\end{tabular}




\begin{tabular}{|c|c|c|c|}
\hline$C(9)-S i(2)-C(6) \# 2$ & $161.1(16)$ & $C(8) \# 1-S i(2)-C(7) \# 2$ & $26(2)$ \\
\hline C(9)\#2-Si(2)-C(6)\#2 & $115.0(15)$ & $C(8)-S i(2)-C(7) \# 2$ & $132.8(11)$ \\
\hline C(9)\#1-Si(2)-C(6)\#2 & $123(2)$ & $C(8) \# 2-S i(2)-C(7) \# 2$ & 108.5(10) \\
\hline$C(6) \# 1-S i(2)-C(6) \# 2$ & $46.2(11)$ & $C(9)-S i(2)-C(7)$ & $110.3(10)$ \\
\hline$C(9)-S i(2)-C(6)$ & $115.0(15)$ & $C(9) \# 2-S i(2)-C(7)$ & $120.6(17)$ \\
\hline$C(9) \# 2-S i(2)-C(6)$ & $123(2)$ & $C(9) \# 1-S i(2)-C(7)$ & $52.6(18)$ \\
\hline$C(9) \# 1-S i(2)-C(6)$ & $161.1(16)$ & $C(6) \# 1-\operatorname{Si}(2)-C(7)$ & 63.4(15) \\
\hline$C(6) \# 1-S i(2)-C(6)$ & $46.2(11)$ & $C(6) \# 2-S i(2)-C(7)$ & 79.5(17) \\
\hline$C(6) \# 2-S i(2)-C(6)$ & $46.2(11)$ & $C(6)-S i(2)-C(7)$ & $108.7(12)$ \\
\hline C(9)-Si(2)-C(8)\#1 & $34(2)$ & $\mathrm{C}(8) \# 1-\mathrm{Si}(2)-\mathrm{C}(7)$ & $132.8(11)$ \\
\hline C(9)\#2-Si(2)-C(8)\#1 & $85(2)$ & $\mathrm{C}(8)-\mathrm{Si}(2)-\mathrm{C}(7)$ & 108.5(10) \\
\hline C(9)\#1-Si(2)-C(8)\#1 & 108.9(10) & $C(8) \# 2-S i(2)-C(7)$ & $26(2)$ \\
\hline C(6)\#1-Si(2)-C(8)\#1 & 105.3(19) & $\mathrm{C}(7) \# 2-\mathrm{Si}(2)-\mathrm{C}(7)$ & $118.7(4)$ \\
\hline C(6)\#2-Si(2)-C(8)\#1 & $128(2)$ & $C(9)-S i(2)-C(7) \# 1$ & $120.6(16)$ \\
\hline$C(6)-S i(2)-C(8) \# 1$ & $82(2)$ & $\mathrm{C}(9) \# 2-\mathrm{Si}(2)-\mathrm{C}(7) \# 1$ & $52.6(18)$ \\
\hline$C(9)-S i(2)-C(8)$ & $108.9(10)$ & C(9)\#1-Si(2)-C(7)\#1 & $110.3(10$ \\
\hline $\mathrm{C}(9) \# 2-\mathrm{Si}(2)-\mathrm{C}(8)$ & $34(2)$ & $C(6) \# 1-S i(2)-C(7) \# 1$ & $108.7(12$ \\
\hline$C(9) \# 1-S i(2)-C(8)$ & $85(2)$ & $C(6) \# 2-S i(2)-C(7) \# 1$ & $63.4(15$ \\
\hline $\mathrm{C}(6) \# 1-\mathrm{Si}(2)-\mathrm{C}(8)$ & 128(2) & $C(6)-S i(2)-C(7) \# 1$ & 79.5(17) \\
\hline$C(6) \# 2-S i(2)-C(8)$ & $82(2)$ & C(8)\#1-Si(2)-C(7)\#1 & 108.5(10) \\
\hline $\mathrm{C}(6)-\mathrm{Si}(2)-\mathrm{C}(8)$ & $105.2(19)$ & $\mathrm{C}(8)-\mathrm{Si}(2)-\mathrm{C}(7) \# 1$ & $26(2)$ \\
\hline $\mathrm{C}(8) \# 1-\mathrm{Si}(2)-\mathrm{C}(8)$ & $112.8(16)$ & C(8)\#2-Si(2)-C(7)\#1 & $132.8(11)$ \\
\hline C(9)-Si(2)-C(8)\#2 & $85(2)$ & $C(7) \# 2-S i(2)-C(7) \# 1$ & $118.7(4)$ \\
\hline C(9)\#2-Si(2)-C(8)\#2 & $108.9(10)$ & $\mathrm{C}(7)-\mathrm{Si}(2)-\mathrm{C}(7) \# 1$ & $118.7(4)$ \\
\hline C(9)\#1-Si(2)-C(8)\#2 & $34(2)$ & $C(8) \# 2-C(7)-C(9) \# 1$ & $37(2)$ \\
\hline $\mathrm{C}(6) \# 1-\mathrm{Si}(2)-\mathrm{C}(8) \# 2$ & 82(2) & C(8)\#2-C(7)-Si(2) & $78.4(17$ \\
\hline$C(6) \# 2-S i(2)-C(8) \# 2$ & $105.2(19)$ & $C(9) \# 1-C(7)-\operatorname{Si}(2)$ & 63.6(11 \\
\hline$C(6)-S i(2)-C(8) \# 2$ & $128(2)$ & $C(8) \# 2-C(7)-C(6) \# 1$ & $117(2)$ \\
\hline C(8)\#1-Si(2)-C(8)\#2 & $112.8(16)$ & $C(9) \# 1-C(7)-C(6) \# 1$ & $121.6(14)$ \\
\hline $\mathrm{C}(8)-\mathrm{Si}(2)-\mathrm{C}(8) \# 2$ & $112.8(16)$ & $\mathrm{Si}(2)-C(7)-C(6) \# 1$ & $59.2(1$ \\
\hline$C(9)-S i(2)-C(7) \# 2$ & $52.6(18)$ & $C(7) \# 1-C(8)-C(9) \# 2$ & $116(3)$ \\
\hline C(9)\#2-Si(2)-C(7)\#2 & 110.3(10) & C(7)\#1-C(8)-Si(2) & $75.8(16$ \\
\hline C(9)\#1-Si(2)-C(7)\#2 & $120.6(17)$ & $C(9) \# 2-C(8)-S i(2)$ & $71.7(1$ \\
\hline C(6)\#1-Si(2)-C(7)\#2 & $79.5(17)$ & C(8)\#1-C(9)-C(7)\#2 & $27(3)$ \\
\hline $\mathrm{C}(6) \# 2-\mathrm{Si}(2)-\mathrm{C}(7) \# 2$ & $108.7(12)$ & C(8)\#1-C(9)-Si(2) & $74.1(1$ \\
\hline$C(6)-S i(2)-C(7) \# 2$ & $63.4(15)$ & $C(7) \# 2-C(9)-S i(2)$ & $63.8(1$ \\
\hline
\end{tabular}

Symmetry transformations used to generate equivalent atoms:

$\# 1-y+1, x-y+1, z \quad \# 2-x+y,-x+1, z$ 
Table S4. Anisotropic displacement parameters $\left(\AA^{2} \times 10^{3}\right)$ for $\mathbf{1 b}$. The anisotropic displacement factor exponent takes the form: $-2 \pi^{2}\left[h^{2} a^{* 2} U^{11}+\ldots+2 h k a * b * U^{12}\right]$

\begin{tabular}{lcccccc}
\hline & $\mathrm{U}^{11}$ & $\mathrm{U}^{22}$ & $\mathrm{U}^{33}$ & $\mathrm{U}^{23}$ & $\mathrm{U}^{13}$ & $\mathrm{U}^{12}$ \\
\hline $\mathrm{W}(1)$ & $73(1)$ & $73(1)$ & $31(1)$ & 0 & 0 & $36(1)$ \\
$\mathrm{P}(1)$ & $78(2)$ & $78(2)$ & $34(2)$ & 0 & 0 & $39(1)$ \\
$\mathrm{C}(2)$ & $93(6)$ & $87(7)$ & $55(9)$ & $45(8)$ & $2(6)$ & $62(5)$ \\
$\mathrm{Si}(1)$ & $80(2)$ & $26(1)$ & $76(2)$ & $8(3)$ & $-37(3)$ & $16(2)$ \\
$\mathrm{C}(3)$ & $76(12)$ & $180(20)$ & $82(8)$ & $27(13)$ & $5(9)$ & $78(15)$ \\
$\mathrm{C}(4)$ & $121(15)$ & $55(10)$ & $85(9)$ & $3(8)$ & $-31(13)$ & $-4(11)$ \\
$\mathrm{C}(5)$ & $76(9)$ & $77(9)$ & $198(18)$ & $-20(13)$ & $45(19)$ & $48(8)$ \\
$\mathrm{C}(6)$ & $87(19)$ & $110(30)$ & $45(7)$ & $18(19)$ & $8(9)$ & $80(20)$ \\
$\mathrm{Si}(2)$ & $78(2)$ & $78(2)$ & $38(3)$ & 0 & 0 & $39(1)$ \\
& & & & & \\
\hline
\end{tabular}


Table S5. Hydrogen coordinates $\left(\times 10^{4}\right)$ and isotropic displacement parameters $\left(\AA^{2} \times\right.$ $10^{3}$ ) for $\mathbf{1 b .}$

\begin{tabular}{lrrrr}
\hline & $x$ & $y$ & $\mathrm{z}$ & $\mathrm{U}(\mathrm{eq})$ \\
\hline $\mathrm{H}(1 \mathrm{~A})$ & 3397 & 8116 & 12635 & 156 \\
$\mathrm{H}(1 \mathrm{~B})$ & 3699 & 8773 & 11443 & 156 \\
$\mathrm{H}(1 \mathrm{C})$ & 4779 & 8653 & 12100 & 156 \\
$\mathrm{H}(2 \mathrm{~A})$ & 5003 & 8785 & 8811 & 85 \\
$\mathrm{H}(2 \mathrm{~B})$ & 5032 & 8509 & 10076 & 85 \\
$\mathrm{H}(3 \mathrm{~A})$ & 7363 & 10153 & 7871 & 158 \\
$\mathrm{H}(3 \mathrm{~B})$ & 7028 & 8856 & 7254 & 158 \\
$\mathrm{H}(3 \mathrm{C})$ & 8353 & 9647 & 7894 & 158 \\
$\mathrm{H}(4 \mathrm{~A})$ & 8000 & 10346 & 10240 & 162 \\
$\mathrm{H}(4 \mathrm{~B})$ & 8349 & 9269 & 10525 & 162 \\
$\mathrm{H}(4 \mathrm{C})$ & 7105 & 9200 & 11048 & 162 \\
$\mathrm{H}(5 \mathrm{~A})$ & 6194 & 6416 & 8552 & 169 \\
$\mathrm{H}(5 \mathrm{~B})$ & 6423 & 6510 & 9859 & 169 \\
$\mathrm{H}(5 \mathrm{C})$ & 7612 & 7144 & 9034 & 169 \\
$\mathrm{H}(6 \mathrm{~A})$ & 2243 & 7073 & 7676 & 83 \\
$\mathrm{H}(6 \mathrm{~B})$ & 1809 & 5653 & 7676 & 83 \\
$\mathrm{H}(7 \mathrm{~A})$ & 4523 & 5633 & 6746 & 58 \\
$\mathrm{H}(7 \mathrm{~B})$ & 5138 & 6583 & 5757 & 58 \\
$\mathrm{H}(7 \mathrm{C})$ & 5307 & 7103 & 6967 & 58 \\
$\mathrm{H}(8 \mathrm{~A})$ & 4268 & 8463 & 5139 & 443 \\
$\mathrm{H}(8 \mathrm{~B})$ & 3151 & 8419 & 5825 & 443 \\
$\mathrm{H}(8 \mathrm{C})$ & 4513 & 9002 & 6346 & 443 \\
$\mathrm{H}(9 \mathrm{~A})$ & 2062 & 4766 & 5479 & 143 \\
$\mathrm{H}(9 \mathrm{~B})$ & 1511 & 5641 & 5131 & 143 \\
$\mathrm{H}(9 \mathrm{C})$ & 2665 & 5711 & 4486 & 143 \\
& & & & \\
\hline
\end{tabular}

